\title{
Harnessing the potential of ligninolytic enzymes for lignocellulosic biomass pretreatment
}

\begin{abstract}
Abundant lignocellulosic biomass from various industries provides a great potential feedstock for the production of value-added products such as biofuel, animal feed, and paper pulping. However, low yield of sugar obtained from lignocellulosic hydrolysate is usually due to the presence of lignin that acts as a protective barrier for cellulose and thus restricts the accessibility of the enzyme to work on the cellulosic component. This review focuses on the significance of biological pretreatment specifically using ligninolytic enzymes as an alternative method apart from the conventional physical and chemical pretreatment. Different modes of biological pretreatment are discussed in this paper which is based on (i) fungal pretreatment where fungi mycelia colonise and directly attack the substrate by releasing ligninolytic enzymes and (ii) enzymatic pretreatment using ligninolytic enzymes to counter the drawbacks of fungal pretreatment. This review also discusses the important factors of biological pretreatment using ligninolytic enzymes such as nature of the lignocellulosic biomass, $\mathrm{pH}$, temperature, presence of mediator, oxygen, and surfactant during the biodelignification process.
\end{abstract}

Keyword: Biological pretreatment; Ligninolytic enzymes; Laccase; Lignocellulosic biomass; Mediator; Lignin degradation 